\title{
Bilateral Periprosthetic Knee Fracture with the Right Floating Total Knee and Left Periprosthetic Patella Fracture Management Strategy: A Case Report
}

\author{
Lavindra Tomar ${ }^{1}$, Gaurav Govil ${ }^{1}$, Pawan Dhawan ${ }^{1}$ \\ Learning Point of the Article:
}

Periprosthetic knee fracture with floating total knee is a rare complex injury which needs individualized early definitive intervention for optimal functional outcome

\section{Abstract}

Introduction: Periprosthetic fractures (PPFs) in total knee replacement are an uncommon condition. The floating knee injury around total knee arthroplasty (TKA) is even rare and poses challenges in management. Incidence is increasing due to growing primary joint arthroplasties and revision procedures. We report a case of bilateral PPF with a floating total knee.

Case Report: A 74-year-old female involved in a violent car accident sustained bilateral knee injuries, facial, and hand injury. In the emergency room, the initial resuscitation and trauma protocol stabilization were done and she was provisionally immobilized for her limb injuries. She presented with the right-sided floating total knee involving periprosthetic periarticular comminuted distal femur fracture and midshaft comminuted fracture tibia fibula. The patient also had left knee lower pole periprosthetic patellar fracture. The patient had a history of bilateral TKA around 2 years back. She underwent surgical management of the right floating total knee by stabilization of distal femur fracture and tibial shaft fracture fixation with locking plates. She underwent primary autologous bone grafting for both fracture sites. The left knee patellar fracture was managed conservatively in a brace. At 8 months follow-up, the patient was pain free and had consolidation of fractures. The patient walked without any walking aids. At 18 months, she had regained her pre-injury functional status.

Conclusion: Each fracture in a floating total knee injury is unique and treatment should be decided based on individual analysis and the extent of soft-tissue injuries. An uncommon occurrence highlights the complex injury patterns involving PPF requiring individualized case specific management strategy.

Keywords: Floating total knee, Periprosthetic knee fracture, floating knee.

\section{Introduction}

There is a global increase in periprosthetic fracture (PPF) incidence due to growing primary joint arthroplasties and revision procedures. An increasing life expectancy, healthy lifestyles, and more active elderlies are becoming common [2, 7]. PPFs can occur in the femur, tibia, or patella, affecting the areas within $15 \mathrm{~cm}$ from the joint surface or within $5 \mathrm{~cm}$ from the intramedullary stem $[2,4,7]$. Periprosthetic femoral fractures above total knee replacement (TKR) are an uncommon condition $(0.3-3 \%)[1,2,4,7]$. Incidence of fracture in the tibia is even more uncommon $(0.1-0.4 \%$ of patients) [4, 7]. Incidence of patellar fractures has been reported as $0.2-21 \%$ in the patella resurfaced knee and $0.05 \%$ in the non-resurfaced knee $[2,4,7]$. Low-energy trauma is most common mechanism of injury though in 10\% high-energy trauma may be the causative factor $[3,4]$. The floating knee injury (FKI) around total knee arthroplasty (TKA) is a rare and challenging scenario. It is usually associated with injuries to head, chest, abdomen, and limbs with overall death rate on admission up to $10 \%$ [5]. Despite all efforts to understand and treat these patients, high rate of complications (50\%) and mortality are still reported [3]. Very few case reports are

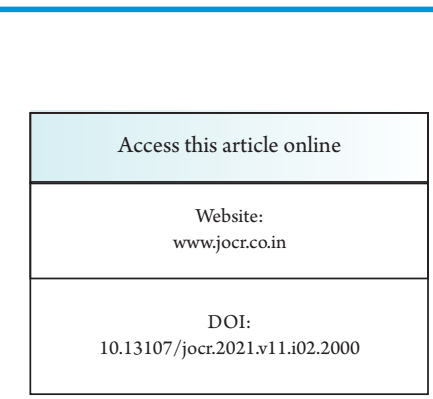

Author's Photo Gallery

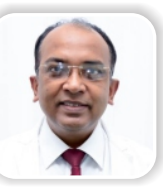

Dr. Lavindra Toma

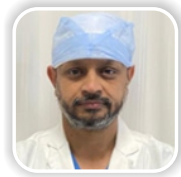

Dr. Gaurav Govil

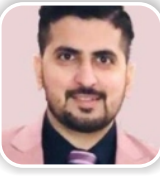

Dr. Pawan Dhawan

${ }^{1}$ Department of Orthopaedics, Max Super Speciality Hospital, 108 A, I.P. Extension, Patparganj, New Delhi, India.

Address of Correspondence:

Dr. Gaurav Govil,

D 101, Sunshine Helios, Sector 78, Noida, Uttar Pradesh - 201305, India.

E-mail: gauravgovil@yahoo.co.in

Journal of Orthopaedic Case Reports | pISSN 2250-0685 | eISSN 2321-3817 | Available on www.jocr.co.in | doi:10.13107/jocr.2021.v11.i02.2000 This is an Open Access article distributed under the terms of the Creative Commons Attribution Non-Commercial License (http://creativecommons.org/licenses/by-nc/3.0) which permits unrestricted non-commercial use, distribution, and reproduction in any medium, provided the original work is properly cited. 


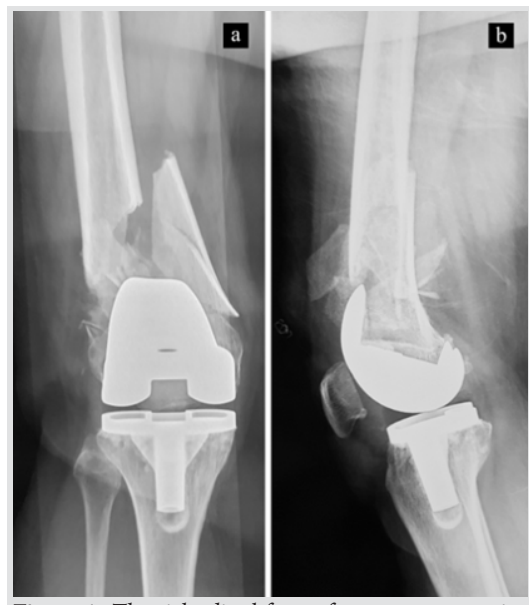

Figure 1: The right distal femur fracture pre-operative anteroposterior view (a) and lateral view (b).

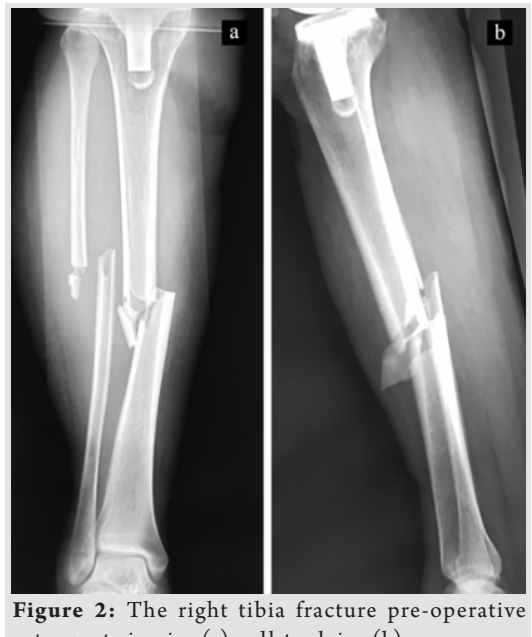

anteroposterior view (a) and lateral view (b).

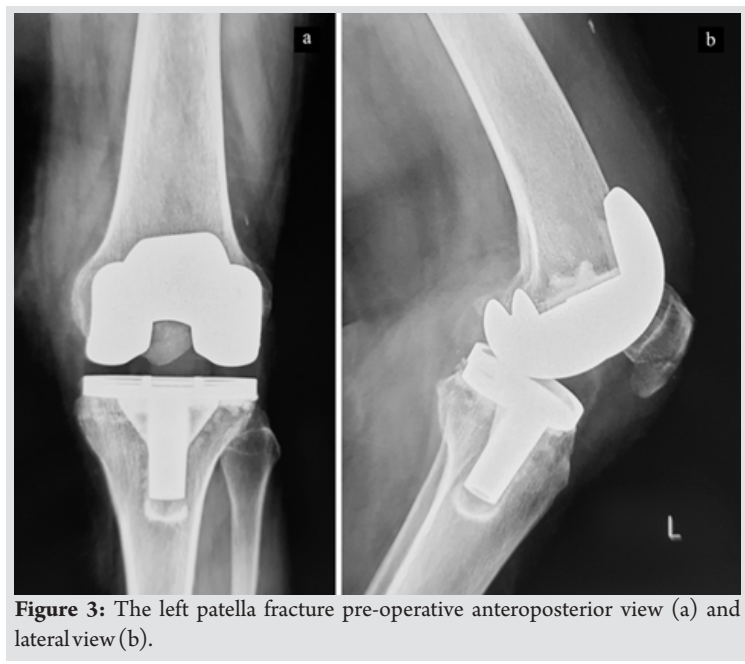

available in literature to delineate treatment algorithm or management strategy for such complex trauma $[6,8]$. We report this case where PPF management of femur, tibia, and patella treatment is considered and managed in a single patient.

\section{Case Report}

A 74-year-old female with a history of road traffic accident presented in the emergency room around $3 \mathrm{~h}$ after sustaining trauma. The patient was a rear seat passenger in a car with a history of loss of life to driver and critical head injury to nondriving front seat copassenger. The patient had bilateral lower limb, facial, and hand trauma. The patient had a history of bilateral TKR (TKA) done 2 years ago. The patient had a history of systemic hypertension on regular treatment. The patient was assessed by emergency physician and initiation of necessary measures including provisional stabilization of lower limbs, fluid replacement, and critical care monitoring of parameters done. The patient had bilateral knee injuries with crepitus, abnormal mobility, and deformity of the right knee and leg. The left knee revealed tenderness and swelling along the joint. There was no sign of neurovascular injury to lower limbs. Additional injuries along face and right hand were softtissue injuries. Plain film radiography showed distal femur comminuted displaced PPF right knee (Fig. 1a, b) classified as type II in accordance with Rorabeck and Taylor criteria [4] and midshaft comminuted fracture right tibia fibula (Fig. 2a, b) classified as type III A in accordance with Felix et al. [4]. The left knee radiography showed undisplaced periprosthetic lower pole fracture of patella (Fig. 3a, b) classified as type IA in accordance with Ortiguera and Berry criteria [4]. The floating knee scenario for the right lower limb was classified as Fraser's type IIb and planned for surgical management for the right lower limb injury.

The patient was stabilized and on the 2 nd day of injury, the patient underwent surgical reduction and fixation of distal femur fracture with distal femoral locking plate (AO Synthes)

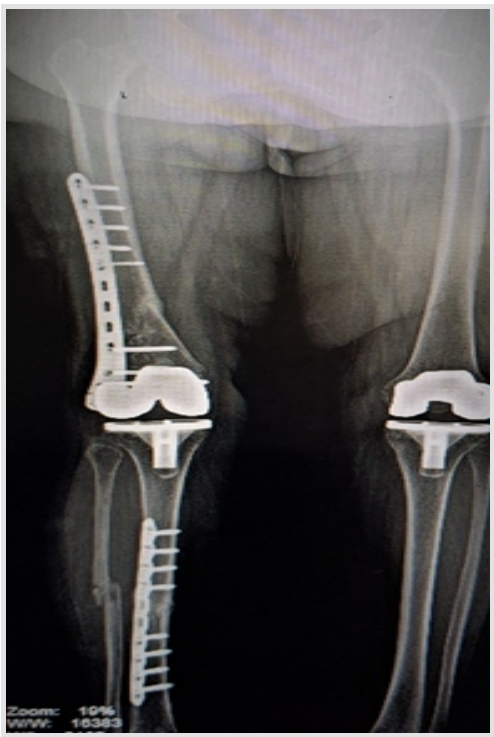

Figure 4: Follow-up scanogram showed good alignment.
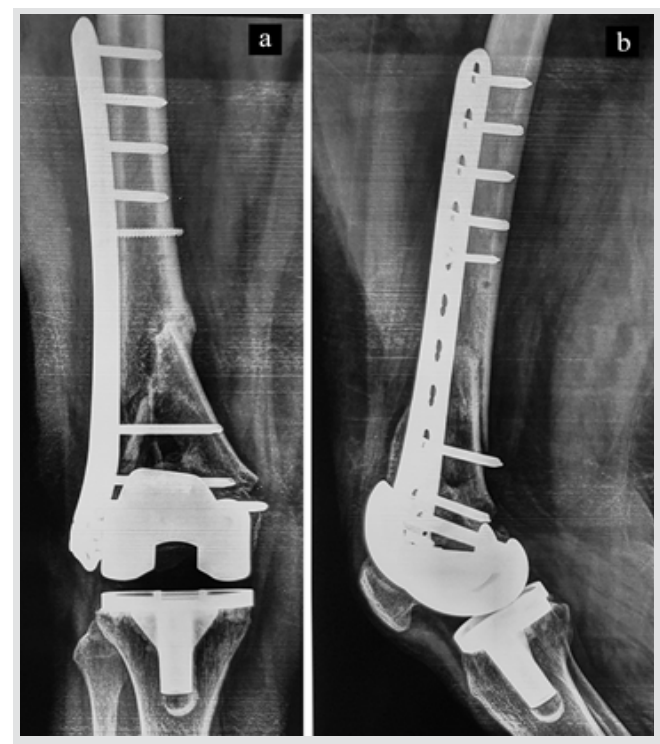

Figure 5: The right femur post-operative anteroposterior view (a) and lateral view (b).
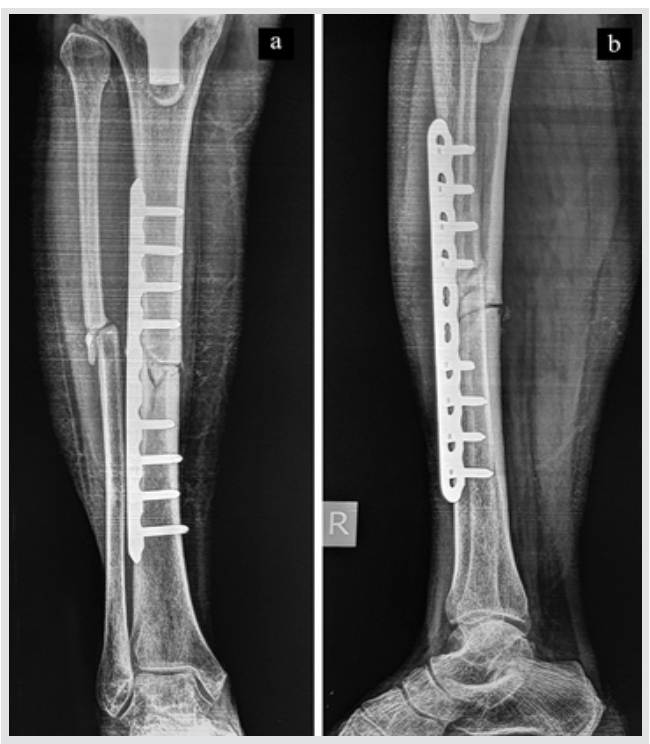

Figure 6: The right tibia post-operative anteroposterior view (a) and lateral view (b). 


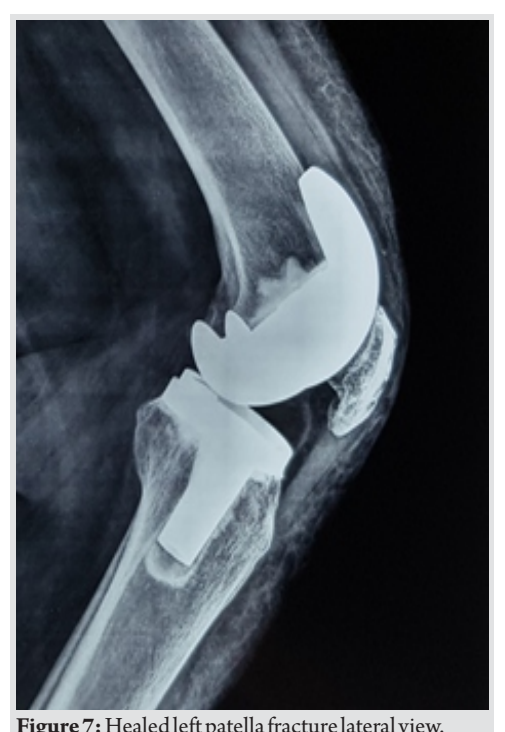

Figure 7: Healed left patella fracture lateral view.

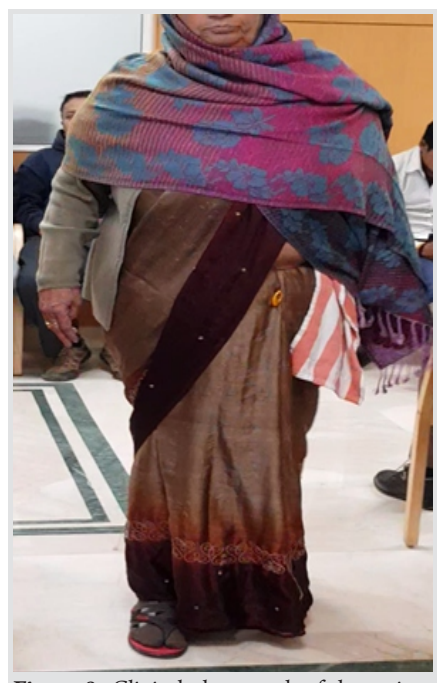

Figure 8: Clinical photograph of the patient standing without support. through the lateral approach and diaphyseal tibial fracture fixation with narrow locking plate (AO Synthes). Primary autologous iliac ipsilateral bone grafting to augment the healing at both fracture segments done. The left knee patellar fracture was managed conservatively in a long knee brace immobilization. Immediate post-operative period was uneventful and the patient was discharged from hospital in stable condition after 5 days of surgery.

Rehabilitation protocol was initiated early with in bed limb exercises and assisted passive range of movements exercises for the right knee and ankle from the 1st week onward. Three weeks after surgery, the patient was allowed walker support walking and rehabilitation of the left knee in a brace support continued. At 6 weeks, the patient was allowed knee mobilization exercise for both knees. The patient was monitored with clinical and radiological assessment at 6,12 , and 24 weeks. At 6 months, the patient was able to mobilize with stick support once there were clear radiologic signs of fracture consolidation. Follow-up scanogram (Fig. 4), radiographs of femur (Fig. 5a, b), tibia (Fig. 6a, b), and patella (Fig. 7) showed good alignment. The patient walked without any walking aids (Fig. 8) with good functional range of movements (Fig. 9a, b) at 18 months follow-up and had recovered her previous functional status.

\section{Discussion}

In 1975, Blake and McBryde established the concept of the "floating knee" to describe homolateral fractures of the femur and tibia, where the knee is disconnected from the rest of the limb [5]. McBryde Type II (29\%) is a variant in which one or both fractures involve the knee [5]. In 1978, Fraser further subclassified floating knee type II injuries to Type II a (8\%) as a tibia plateau fracture associated with a femoral shaft fracture, type II b (12\%) as an articular fracture of distal femur associated

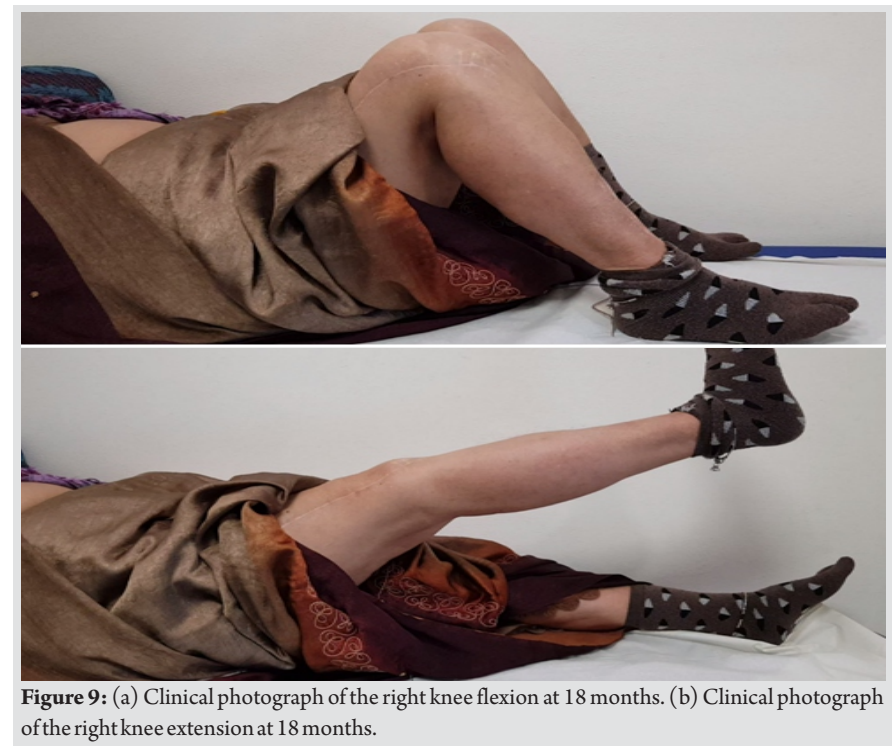

with a tibial shaft fracture, and type II c (9\%) as a fracture of the tibia plateau and articular fracture of the distal femur [5]. The "floating total knee" is first described in literature by Jeong et al. in 2006 [6]. In 2011, Loubignac et al. reported a highly unusual case of a young adult presenting with polytrauma involving bilateral floating knee. On literature search, a bilateral PPF presenting with a "floating total knee" on the right lower limb and a patellar fracture on the left lower limb is not yet described.

PPF is a major challenge to orthopedic surgeons [2], with a large variety of implants, designs, concepts, and principles needed to be considered for each patient, on an individual basis for its management [1]. This type of fractures, commonly seen in older patients, is often caused by minor trauma such as a fall from standing height in $90 \%$ and less frequently by high-energy trauma (road traffic accidents, seizures, or forced manipulation of a stiff knee) [1]. The late PPFs occur mostly within 2-5 years after primary surgery [2,9]. Jeong et al. (2006) reported a lowenergy trauma in a rheumatoid patient on prolonged steroid intake as a predisposing factor after 3 years of the primary TKA [6]. Our patient has high-energy trauma sustained in a road traffic accident with concurrent critical injuries to copassengers at 2 years post-TKA.

Risk factors for these PPFs include patient specific factors such as elderly, female sex, osteoporosis, a history of revision arthroplasty, chronic steroid therapy, rheumatoid arthritis, and neurologic disorders [1, 3, 4]. Radiographic osteolysis, infection, and navigation-assisted arthroplasty are also considered as pre-disposer to fracture [4]. Technique-related factors like anterior femoral notching are a risk factor for supracondylar fracture femur $[3,4]$. Although notching is implicated, larger series do not show any clear association [4]. Our patient is elderly female though without any other specific risk factor or femoral notching as a causative factor.

The classification of periprosthetic supracondylar fractures by 
Rorabeck is commonly used and takes into account the displacement of the fracture and the stability of the prosthesis [7]. Felix et al. classified periprosthetic tibial fractures into four types based on the anatomic location and proximity to the prosthesis as well as the status of the prosthetic fixation [7]. Ortiguera and Berry classification of patellar fractures following TKA consists of four types and takes into account the stability of the implant fixation, the status of the extensor mechanism, the location of the fracture, and adequacy of the bone stock [4]. Faukler et al. established an implant-dependent classification for PPF of the distal femur relating to the most common types of prosthesis with four fracture types created and defined in 2017 [12]. However, no classification for a periprosthetic floating total knee is available in the literature.

PPFs involving both the distal femur and proximal tibia have been documented in case reports and management detailed case wise. The long period of immobilization, struggle to maintain fracture reduction, loss of knee range of motion, malalignment, and risk of non-union make conservative treatment for displaced PPF scarcely appealing [8]. If the implant is stable, immobilization is unlikely to maintain reduction of a fracture, surgery is appropriate. The purpose of the surgical procedure in this type of fractures is to preserve limb length, restore rotational alignment, and allow early motion $[1,3]$.

The quality of the periprosthetic bone is often poor as a result of stress shielding from the prosthesis and because of age-related osteoporosis in typically older population with TKAs [4]. Poor bone stock, pre-existing implant, and bone cement may impede fracture reduction and fixation, predisposing to non-union or malunion $[2,4]$.

A periprosthetic distal femur fracture surgical management includes locking or non-locking plate fixation, utilizing direct or indirect reduction, or retrograde intramedullary nailing (RIMN) $[4,7]$. Carvalho et al. advocated use of relative stability with a distal intramedullary technique to achieve indirect bone healing by preserving callus induction potential [1]. Cavanellas et al. used polyaxial locking plate fixation of distal femoral fracture in view of distal fracture fragment being $<2 \mathrm{~cm}$ size probably insufficient to hold with RIMN [3]. Herrera et al.., after a systematic review, showed no advantage between these two fixation techniques [3]. Jeong et al. used highly constrained cemented revision TKA for management [6]. Different reduction techniques, stability principles, and arthroplasty options have been considered to treat such fractures. We did a distal femur locking plate fixation for stabilization.

Advantages of locking compression plates in knee periprosthetic femur fracture setting are minimal invasiveness, strength of fixation in osteoporotic bone, and the possibility of monocortical fixation coupled with metal cerclage in proximity to the prosthetic implant [8]. For intramedullary nailing, it is important to note the type of knee arthroplasty implant used. Some closed-box posterior stabilized femoral component designs may not allow this technique $[1,4]$. Revision TKA in unstable implant with poor soft tissue or vascular perfusion may delay wound healing and increase risk of infection and necrosis [2].

Non-displaced fractures extending into the tibial plateau (Felix type IA) are usually stable and can be treated non-surgically with protected weight-bearing and bracing or casting [4]. Most displaced type IIA, IIIA, and IVA fracture are best treated with internal fixation, typically locking plate and screw fixation [4]. When the tibial component of a TKA is loose (Felix types IB, IIB, IIIB, and IVB fractures, revision arthroplasty with a longstemmed tibial component is best [4]. In our case, tibial fracture is managed with locking plate fixation.

Patellar fractures can occur due to direct trauma or fatigue [2]. Conservative treatment can be considered as a treatment option in the absence of disruption of the extensor mechanism and patellar component instability [2, 4, 7]. Bazylewicz and Egol suggested non-surgical management with partial weightbearing in a knee immobilizing device for 6-8 weeks [4]. Fractures associated with disruption of the extensor mechanism are usually treated operatively, regardless of the stability of the patellar component [9]. Loosening of the patellar component requires surgical intervention for the removal of the loose implant and patelloplasty with good bone stock or patellar resection arthroplasty with partial or total patellectomy in bone deficient injuries. Considering the stable patellar fracture pattern, conservative management was considered for the left PPF management in our patient.

Acute bone grafting for PPFs of the femur is controversial and probably unnecessary in most patients if softtissue disruption is minimized and bone contact is maintained. The decision to use acute bone grafting may be made on a casebycase basis [4]. Since non-union is a concern in a floating segmental bony injury, we did primary autologous bone grafting to allow a more biological environment for healing.

The functional assessment after the treatment of FKIs is evaluated by most authors using the Karlström and Olerud grading system [5]. At 18 months, the patient has regained preinjury functional status. She has no pain in bilateral knees and no shortening, rotational deformity, or restricted range of movements. The patient is graded with an excellent outcome.

\section{Conclusion}

Each fracture in a floating total knee injury is unique and treatment should be decided based on individual analysis and 
the extent of soft-tissue injuries [5]. The treatment is "more experience than evidence based" [5]. The chosen method depends on the fracture pattern, location, soft-tissue injury whether open or closed, available resources, surgical capability, and preference [5]. A satisfactory outcome is possible with timely intervention and adherence to basic osteosynthesis surgical principles. Reporting of such injury pattern in literature will enhance better understanding of management strategy for such floating total knee injury.

\section{Clinical Message}

Floating total knee is an uncommon rare condition which needs to be reported for understanding of management concerns.

\section{References}

1. Carvalho M, Fonseca R, Simões P, Bahute A, Mendonça A, Fonseca F. Bilateral distal femoral nailing in a rare symmetrical periprosthetic knee fracture. Case Rep Orthop 2014;2014:745083.

2. YooJD, Kim NK. Periprosthetic fractures following total knee arthroplasty. Knee Surg Relat Res 2015;27:1-9.

3. Loures FB, Motta JR, Albuquerque RS, Barretto JM, Cavanellas NT. Bilateral distal femoral fracture after total knee arthroplasty. Rev Bras Ortop 2016;51:606-9.

4. Bazylewicz DB, Egol KA. Periprosthetic fractures after total knee arthroplasty. Orthop Knowl Online J 2014;12:???. A v a i 1 a b 1 e f $\mathrm{r}$ o $\mathrm{m}$ : http://www.orthoportal.aaos.org/oko/article.aspx?

5. Vives JM, Bel JC, Agundez AC, Rodríguez FC, Traver JP, Schultz-Larsen $\mathrm{M}$, et al. The floating knee: A review on ipsilateral femoral and tibial fractures. EFORT Open Rev 2016; 1:375-82.

6. Jeong GK, Pettrone SK, Liporace FA, Meere PA. Floating total knee: Ipsilateral periprosthetic fractures of the distal

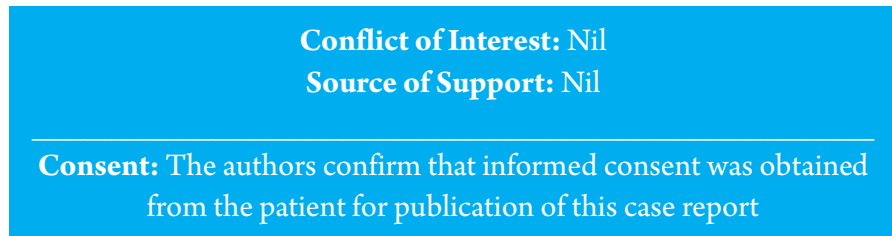

femur and proximal tibia after total knee arthroplasty. J Arthroplasty 2006;21:138-40.

7. Gordon MC, Khan W, Johnstone D. Recent advances and developments in knee surgery: Principles of periprosthetic knee fracture management. Open OrthopJ 2012;6:301-4.

8. Canton G, Ratti C, Fattori R, Hoxhaj B, Murena L. Periprosthetic knee fractures. A review of epidemiology, risk factors, diagnosis, management and outcome. Acta Biomed 2017;88:118-28.

9. Marya SK, Kacker S, Singh C. Periprosthetic fractures-a review article.J Clin Orthop Trauma 2011;2:3-11.

10. Kumar R. The floating knee injury. J Clin Orthop Trauma 2011;2:69-76.

11. Loubignac F, Pernin C, Buord JM. Bilateral floating knee: An exceptional injury. InjExtra 2011;42:17-21.

12. Fakler JK, Pönick C, Edel M, Möbius R, Brand AG, Roth A, et al. A new classification of TKA periprosthetic femur fractures considering the implant type. BMC MusculoskeletDisord 2017;18:490.
How to Cite this Article

Tomar L, Govil G, Dhawan P. Bilateral Periprosthetic Knee Fracture with the Right Floating Total Knee and Left Periprosthetic Patella Fracture Management Strategy: A Case Report. Journal of Orthopaedic Case Reports 2021 February; 11(2): 1-5. 